\title{
Diseño de un pozo geonavegado usando sistemas rotativos direccionales híbridos
}

\section{Design of a oliwell using rotative directional hybrid systems}

\author{
págs. 82-90
}

Grupo de Investigación: Nuevas tecnologías de perforación (NUPETER)

Línea de investigación: Nuevas tecnologías de perforación

Jaime A. Polanco J. \& Andrés L. Ramírez D.*

Recibido: 2 de febrero de 2016 Aceptado: 25 de abril de 2016

\section{Resumen}

El Campo Castilla de Ecopetrol S.A, los últimos años, se han presentado altos Tiempos No Planeados (NPT'S), generando sobre costos en la perforación. La tecnologia de Sistemas Rotativos Direccionales (RSS) ha sido usada para solucionar los problemas en la perforación de pozos direccionales. Este artículo mostrará el diseño de un pozo geonavegado usando RSS Híbridos (HRSS) que involucran la tecnología Push \& Point-the-Bit, por medio de la unidad productora de la Formación K1, en el Campo Castilla, logrando reducir 4 días de perforación respecto al mejor pozo perforado de la muestra, con un ahorro de 320 mil dólares. El diseño del pozo se ha realizado 5 en etapas, que incluyen: Investigación tecnológica, diseño de la trayectoria, diseño de la sarta, discusión técnica y discusión económica.

Palabras clave: campo castilla, perforación direc." cional, sistemas rotativos direccionales híbridos.

\begin{abstract}
The last years in the oilfield Castilla, have been had numerous problems caused by Non-Productive-Time (NPT), generating higher costs of drilling operations. That's where technology Directional Rotating Systems (RSS) can solve problems evidenced during the drilling of directional wells in the Oilfield Castilla. Has considered designing a lateral well using Hybrid Rotary Steerable Systems (Hybrid RSS) involving the Push-The-Bit \& Point-The-Bit Bit, less drilling time and at lower cost.

This article will show the design of a well to the K1 lateral navegation Training in the Oilfield Castilla Ecopetrol S.A. reducing drilling four days about the best studied well which was performed in 15.6 days at a savings of up to $\$ 320,000$. Well design has been carried out in 5 steps, technology research, trajectory design, BHA design, technical discussion and economical discussion.
\end{abstract}

Keywords: castilla field, directional drilling, hybrfid directional rotative systems.

- Ingeniero de petróleos, coinvestigador. Fundación Universidad de América. japj182@hotmail.com

-• Ingeniero de petróleos, coinvestigador. Fundación Universidad de América. andres_radu@hotmail.com 
LÍNEA DE INVESTIGACIÓN: NUEVAS TECNOLOGÍAS DE PERFORACIÓN

\section{Introducción}

Los Sistemas Rotativos Direccionales fueron por primera vez introducidos al mercado en 1996, Los (RSS) incluyen una unidad de orientación a unos cuantos ft de la broca, sensores de mediciones mientras se perfora para el monitoreo de la dirección del pozo y un sistema de comunicación entre la superficie y el fondo para mantener la dirección deseada dando comando al sistema.

Su funcionamiento se destaca a diferencia de los motores de fondo de perforación en que el motor y la sarta de perforación rotan con la broca evitando errores humanos al definir y cuidar la trayectoria, tiempos de viajes, pega de tuberías, facilitando al rotar toda la tubería de perforación, la limpieza del pozo..

Los sistemas rotativos direccionales pueden ser categorizados por su modo de operación: Existen dos conceptos principales por sus sistemas direccionales: Point-The-Bit, y Push-The Bit. La herramienta Push-The-Bit es operada aplicando una fuerza lateral contra la formación en la cara del pozo perforada. Esto puede ser hecho sincronizando las aspas o por una fuerza constante sobre un ensamblaje estacionario.
El sistema Point-The-Bit opera mediante la colocación de una curva en el sistema, esta curva se mantiene geoestacionaria con respecto a la formación. Para entender mejor el principio Point-The-Bit", se puede hacer comparaciones con los sistemas de perforación convencionales que utilizan motores o turbinas donde la broca y la sarta de perforación giran. La rotación de la sarta de perforación niega el efecto de la carcasa doblada, y la broca realiza una trayectoria recta paralela sobre calibre al eje de la sarta de perforación por encima de la carcasa doblada. En el modo de deslizamiento, sólo el rota la broca de perforación.

Modo Push-The-Bit: El sistema de perforación RSS de Push-the-Bit empuja contra la pared del pozo para llevar la sarta de perforación en la dirección deseada. Una versión de este dispositivo de perforación direccional con sistemas rotativos utiliza una unidad de desviación con tres almohadillas de direccionamiento colocadas cerca de la boca para aplicar una fuerza lateral contra la formación (abajo). Para incrementar el ángulo de construcción, cada almohadilla accionada por el lodo empuja contra el lado inferior del pozo a medida que gira hacia su Posición; para disminuir el ángulo, cada almohadilla empuja contra el lado alto (Figura 1).

Figura 1.

Modo Push-the-Bit

Push-the-Bit Dinámico

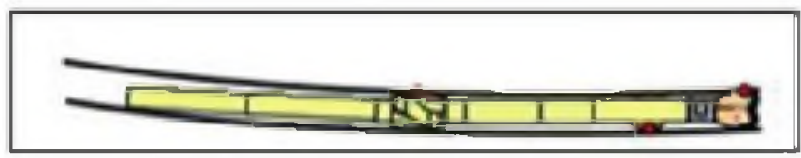

Push-the-Bit Estático

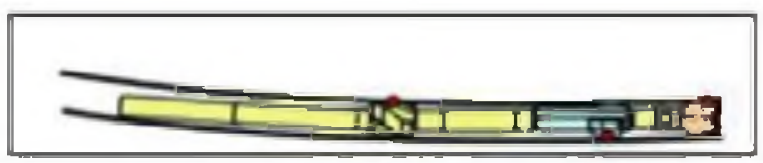

Nota: La Figura 1. Simplifica los modos de perforación usando el sistema Push-The-Bit, el cual, en el modo dinámico usa la interacción de la formación y con una aleta rotaria para generar la dirección según la trayectoria deseada, el modelo estático opera con las aletas estáticas, entre la formación y el cuerpo de direccionamiento.

Fuente: High Dogleg Rotary Steerable System: A Step Change in Drilling Process,", de Conferencia Técnica y Exhibición Anual de la SPE, Nueva Orleans, 4-7 de octubre de 2009

Modo Point-The-Bit: Un sistema Point-theBit utiliza un eje interno para desplazar la alineación entre el eje de la herramienta y el eje del pozo produciendo una respuesta direccional. En un sistema Point-the-Bit, el codo está incluido dentro del collar de la herramienta, inmediatamente arriba de la broca (derecha). Los sistemas Point-the-Bit cambian la trayectoria del pozo 
cambiando el ángulo de orientación de la herramienta. La trayectoria cambia en la dirección del codo. Esta orientación del codo está controlada por un servomotor que gira a la misma velocidad que la sarta de perforación, pero en sentido contrario. Esto permite que el ángulo de orientación de la herramienta permanezca geoestacionario, o sin rotar, mientras que el collar gira.

Figura 2.

Modo Point-the-Bit

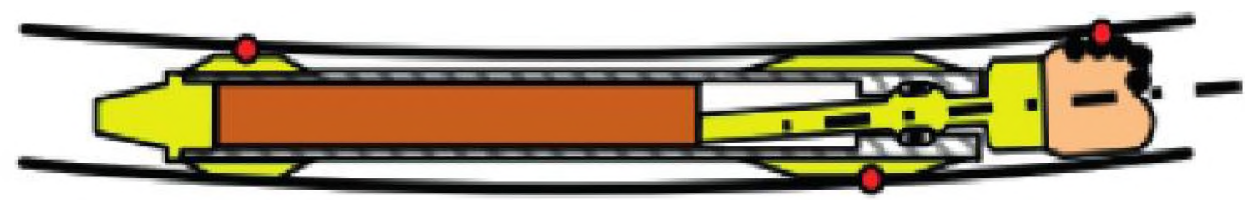

Nota: El modo Point-The-Bit como lo muestra la figura 2 usa en su unidad de direccionamiento un motor que direcciona la broca en su punto de pívot, hacia la trayectoria deseada de perforación.

Fuente: AADE-08-DF-HO-39, 2009 pág., 3.

\section{Sistemas Rotativos Direccionales Híbridos}

Según los autores: Carrizo, Hector R; Sanchez, Jose L; Perdomo, Luis; Bertarelli, Mario. Definen los sistemas rotativos direccionales como:

"Esta combinación hibrida de sistemas "Push-The-Bit" y

Point-The-Bit" son reflejados en una herramienta que incrementa la capacidad de alcanzar altas severidades de patadas de perro (DLS) sin comprometer la velocidad de penetración. Todos los componentes externos que forman parte de esta herramienta giran con la sarta de perforación, lo que permite una mejor limpieza del pozo al tiempo que reduce el riesgo de quedarse atascado. En pruebas de Campo, la herramienta ha logrado una tasa de acumulación de $17^{\circ} / 100 \mathrm{ft}$ que hace que sea una excelente herramienta para pozos horizontales de mediano radio"... Traducción propia. Fragmento artículo SPE-169295-MS

En este ensamble Figura 3. Se evidencia el mecanismo de direccionamiento, común en un Sistema Rotativo Direccional Híbrido, en la sección de direccionamiento muestra los dos principios Push, y Point the Bit, en la parte superior se muestran en círculo rojo la sección que ejerce la operación de empuje contra la manga de direccionamiento, activada por acción del lodo, enseguida se muestra como luego de accionar el empuje de las almohadillas contra la manga de dirección es activado el pivote de direccionamiento el cual dará continuidad a la trayectoria del pozo con bajo tortuosidad de pozo. En la Figura 4. Muestra la diferencia en un perfil direccional con herramientas direccionales y el sistema híbrido.

\section{Diseño del pozo HRSS-1}

El pozo diseñado fue tomado a partir del mejor pozo seleccionado de la muestra para tomarlo en correlación para el diseño del nuevo pozo con el objetivo de perforar las arenas productoras de la Formación K1 inferior, a continuación se mostrará el diseño del pozo realizado.

\subsection{Trayectoria}

En el diseño de la trayectoria fue considerado, tomando el estudio de anticolisión llevado a cabo con los pozos aledaños a más de $150 \mathrm{Ft}$ de superficie del pozo de estudio. En aquel pozo fue necesario direccionar desde los $400 \mathrm{Ft}$ del pozo para evitar una colisión con el pozo Cast-450, así mismo, a $8400 \mathrm{Ft}-\mathrm{MD}$ de profundidad se encuentra el mayor riesgo de colisión con el pozo Cast220 con un factor de seguridad de 3.9 lo que no representa riesgo alguno para la operación. 
LÍNEA DE INVESTIGACION: NUEVAS TECNOLOGIAS DE PERFORACION

Figura 3.

Ensamblaje Sistema Rotativo Direccional hibrido.

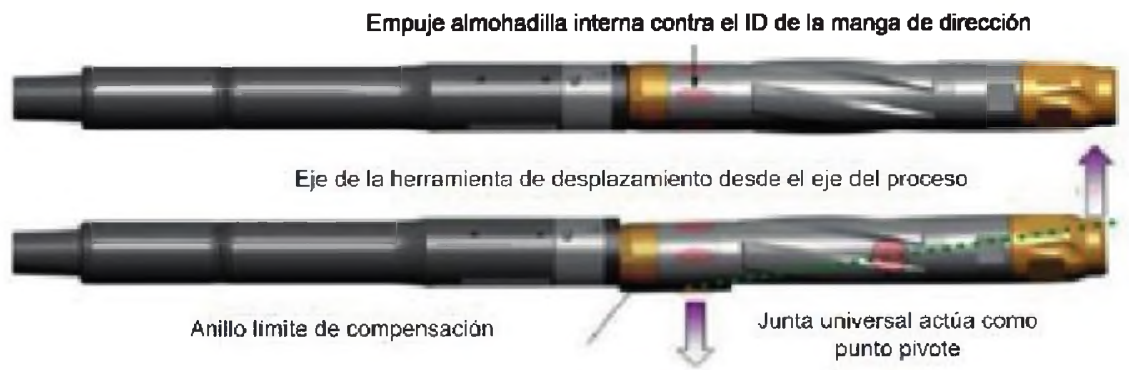

Nota: En este ensamble, se evidencia el mecanismo de direccionamiento, común en un Sistema Rotativo Direccional Híbrido.

Fuente: Albardisi, Tareq; SPE-170019-MS Hybrid High Build 50 Rate RSS Improves Challenging Directional Control in a Soft Abrasive Drilling Environment in Oman Drilling Operation2014.

Figura 5.

Trayectoria pozo HRSS-1

\begin{tabular}{|c|c|c|c|c|c|c|c|c|c|c|c|c|}
\hline $\begin{array}{l}\text { ND } \\
\text { (ft) }\end{array}$ & $\begin{array}{l}\text { Inc } \\
(1)\end{array}$ & Avi & $\begin{array}{l}\text { TVD } \\
\text { (fi) }\end{array}$ & $\begin{array}{l}N / S \\
\text { (ft) }\end{array}$ & $E N$ & $\begin{array}{c}\text { V.Sec } \\
\text { (ft) }\end{array}$ & $\begin{array}{c}\text { Dieg } \\
\text { ("/100ft) }\end{array}$ & $\begin{array}{c}\text { T.Face } \\
(*)\end{array}$ & $\begin{array}{c}\text { Build } \\
\text { (*M00ft) }\end{array}$ & $\begin{array}{c}\text { Tum } \\
\text { (t/100ft) }\end{array}$ & $\begin{array}{c}\text { Section } \\
\text { Type }\end{array}$ & Target \\
\hline 0 & 0 & 0 & 0 & 0 & 0 & 0 & 0 & 0 & 0 & 0 & Tie Line & \\
\hline 400 & 0 & 140 & 400 & 0 & 0 & D & 0 & 140 & 0 & 0 & InCAZI TVD & \\
\hline 986 & 5.86 & 140 & 985 & -22.9 & 19.2 & 6.6 & 1 & 140 & 1 & 0 & Inc Aż TVD & Guayabo \\
\hline 995 & 5.95 & 140 & 993.9 & -23.6 & 19.8 & 8.8 & 1 & 0 & 1 & 0 & Inc Azj MD & Arenisca superior \\
\hline 1200 & 8 & 140 & 1197.4 & -42.7 & 35.8 & 12.3 & 1 & 0 & 1 & 0 & Inc Az] MD & \\
\hline 1800 & 8 & 140 & 1791.6 & -160.7 & 89.5 & 30.8 & 0 & 0 & 0 & 0 & Inc Azi MD & \\
\hline 1980.8 & 8.19 & 140 & 1971 & -123.8 & 103.9 & 35.7 & 1 & 180 & -1 & 0 & Inc Azl MD & Luttta E \\
\hline 2306.8 & 2.93 & 140 & 2295.9 & -143.6 & 120.5 & 41.4 & 1 & 180 & -1 & 0 & Inc Azl DLS & \\
\hline 2599.8 & 0 & 0 & 2588.8 & -149.4 & 125.3 & 43.1 & 1 & 180 & -1 & 0 & IncAzi DLS & \\
\hline 3314 & 0 & 0 & 3303 & -149.4 & 125.3 & 43.1 & 0 & 0 & 0 & 0 & Inc Azi MD & \\
\hline 3383 & 0 & 0 & 3372 & -149.4 & 125.3 & 43.1 & 0 & 0 & 0 & 0 & Inc Azj MD & \\
\hline 5200 & 0 & 0 & 5189 & -149.4 & 125.3 & 43.1 & 0 & 0 & 0 & 0 & IncAzi MD & \\
\hline 6348.6 & 36.11 & 58.54 & 6263 & 33.3 & 424 & 382.3 & 3.14 & 58.54 & 3.14 & 0 & Inc Azi TVD & San Fernando T2 \\
\hline 8422.2 & 38.11 & 58.53 & 6322.5 & 58 & 461 & 435.8 & 0 & 0 & 0 & 0 & Slat & K1 Superior \\
\hline 7058.4 & 87 & 58.53 & 6615.7 & 339.5 & 922.6 & 975.2 & 8 & 0 & 8 & 0 & (ditto) & $\begin{array}{l}\text { K1 inferior - Entry } \\
\text { point }\end{array}$ \\
\hline 7466.3 & 87 & 58.53 & 6637 & 551.1 & 1270 & 1381.4 & 0 & 0 & 0 & 0 & (ditto) & \\
\hline 8700 & 87 & 58.53 & 6701.6 & 1194.3 & 2320.9 & 2610.1 & 0 & -90 & 0 & 0 & Ine Azj MD & \\
\hline
\end{tabular}

Nota: La figura 5, muestra la trayectoria del pozo HRSS-1, el pozo es planeado para realizarse a tasa de construcción de $8 \% 100 \mathrm{Ft}$ en la sección de 8 \% 2 ", lo cual permite profundizar el KOP ahorrando tiempos y costos netos de perforación.

Fuente: Autores mediante Trayectoria COMPAS, Landmark R500.

Anticolisión: El sistema de anticolisión muestra la efectividad del pozo propuesto respecto a los pozos pre existente, como lo muestra la Grafi- ca 1. En él se evidencia la distancia del pozo propuesto a los pozos más cercanos, con los cuales el sistema de medición anticolisión tendrla riesgo. 


\subsection{Ensamblaje}

Para el diseño del pozo Cast-HRSS-1 se toman en cuenta tres ensamblajes, como se muestran a continuación.

Sección 17 1/2"@ 1010 Ft: Para esta sección se sugiere utilizar motor direccional de desplazamiento positivo de $5 / 6$ con el fin de iniciar direccionamiento a los $400 \mathrm{ft}$ MD y no colisionar con la trayectoria del Pozo C 451, por ellos se usó la herramienta MWD cercano a la broca y así asegurar la dirección, sistema MWD de 9 1/2" y una broca PDC de 17 1/2".

Sección 12 1/4"@ 5100 Ft: Para esta sección se sugiere el diseño de BHA para iniciar la sección de 12 1/4", iniciando a 6 grados de inclinación, verticalizando el pozo y luego llevando la trayectoria sin cambios de inclinación y direccionamiento hasta $5093 \mathrm{ft}$, con 0 grados de inclinación y 140 grados de azimuth. Se sugiere utilizar un Sistema Rotativo Direccional (RSS) modo Push-the-Bit con motor de lodo que aporta potencia al RSS, MWD de pulso simple y una Broca PDC de $12 \frac{1 / 4}{4}$, las cuales se prevé tengan la capacidad requerida de construcción, se utiliza el RSS perforando hasta $5100 \mathrm{Ft}$ llegando a al tope de la Formación E3 donde se hace un repaso hasta el zapato de la sección anterior.

Sección 12 1/4"@ 6340 Ft: Para esta sección se sugiere el diseño de un BHA para finalizar la sección de 12 1/4", el propósito de este segundo $\mathrm{BHA}$ análogo al segundo $\mathrm{BHA}$, se debe a un viaje programado a la profundidad de $5100 \mathrm{ft}$, adicionalmente en este BHA se incorpora el uso del modo direccional Push-The-bit, para generar ángulos de construcción de $3 \% 100 f t$ hasta el fin de la sección $12 \frac{1}{4}$ ". Se continua la sección de $121 / 4$ " perforando con RSS hasta el tope de la formación T2 donde se sienta el nuevo zapato a $6340 \mathrm{Ft}$ desde donde iniciara la perforación con el modo direccional Hybrid Rotary Steerable System.
Sección 8 1/2"@8700': Para esta sección se sugiere utilizar en el diseño del BHA un Sistema Rotativo Direccional Híbrido (HRSS), con el fin de conseguir la trayectoria deseada donde es necesario una construcción de $8 \% 100 \mathrm{ft}$, y lograr una Sección Vertical (V. Sec) de $2446 \mathrm{ft}$, con una inclinación de 85 grados a lo largo de $1763 \mathrm{ft}$ navegados, con el propósito de navegar por la arena objetivo (Unidad K1) el tamaño del target fue seleccionado estadísticamente del promedio de navegación de pozos en el Campo Castilla con objetivo la Formación $\mathrm{K} 1$, se adiciono unidades MWD y LWD, una Broca PDC de 8 1/2". Para el BHA de esta sección analizando el torque y arrastre se recomienda la disminución de la cantidad de DC debido a que se observa que generan un arrastre innecesario.

\section{Resultados}

El estudio técnico de ingeniería incluye los trabajos realizados bajo los parámetros de Ecopetrol S.A. en los pozos del Campo Castilla; Ilevado a cabo usando la suit de LandMark, para este caso involucra los resultados dados y simulados bajo el simulador WellPlan de la suit. A continuación se mostraran los resultados de hidráulica, torque y arrastre para cada sección:

\subsection{Sección 17 1/2"@ 1010 Ft}

El estudio de ingeniería demuestra la viabilidad técnica para realizar la sección inicial o de superficie de $171 / 2$ ", en el cual no se supera las limitantes técnicos de operación que se permite para la perforación de un pozo hasta la profundidad de $1010 \mathrm{Ft}$, perforando a una tasa de perforación promedio de $121 \mathrm{Ft} / \mathrm{hr}$, cabe destacar que el campo de petróleo de estudio, usa una bota perforada de $100 \mathrm{Ft}$ con broca triconica, para el estudio se vio no necesario usar este ensamblaje siempre y cuando se mantenga la operación dentro de parámetros. 
LÍNEA DE INVESTIGACIÓN: NUEVAS TECNOLOGÍAS DE PERFORACIÓN

Figura 6.

Ensamblaje sección 17 1⁄2" diseñado para el pozo HRSS-1

\begin{tabular}{|c|c|c|c|c|c|c|c|c|c|}
\hline 4 & 4 & $\begin{array}{c}\text { No. } \\
\text { Ensamblaje }\end{array}$ & $\begin{array}{l}\text { Section } \\
\text { Type }\end{array}$ & $\begin{array}{l}\text { Length } \\
\text { (ft) }\end{array}$ & $\begin{array}{l}\text { Measured } \\
\text { Depth (ft) }\end{array}$ & OD (in) & ID (in) & $\begin{array}{c}\text { Weight } \\
\text { (ppf) }\end{array}$ & Item Description \\
\hline ; & & 1 & Drill Pipe & 488.7 & 488.7 & 5 & 4.275 & 19.5 & $\begin{array}{l}\text { Drill Pipe, } 5 \text { in, } 19.50 \text { ppf, 5, } 4.500 \\
\text { NC50(XH), p }\end{array}$ \\
\hline 7 & & 2 & $\begin{array}{l}\text { Heavy } \\
\text { Weight }\end{array}$ & 231 & 719.7 & 5 & 3 & 49.7 & HWSP, 5 in, 49.70 ppf, 4.500 NC50(XP), P \\
\hline & & 3 & Sub & 3 & 722.7 & 7.68 & 3.24 & 132.3 & Cross Over 6.469 in, 55/8 REG \\
\hline & & 4 & Drill Collar & 186 & 908.7 & 8 & 2.875 & 160.2 & Drill Collar 8 in, 2 in, 6 5/8 REG \\
\hline & & 5 & Sub & 3 & 911.7 & 8.25 & 3.75 & 97.72 & Cross Over, 8.250 in, 97.22 ppf, $65 / 8 \mathrm{FH}$ \\
\hline a & & 6 & MWD & 30 & 941.7 & 8.25 & 2.5 & 97.73 & MWD Tool, 8.250 in, 97.73 ppf, 6 5/8 FH \\
\hline & & 7 & Sub & 3 & 944.7 & 8.25 & 3.75 & 92.73 & Cross Over, 8.250 in, 92.73 ppf, 6 5/8 REG \\
\hline , & & 8 & Drill Collar & 30 & 974.7 & 8 & 2.813 & 158.57 & MMDC 8 in, 2 in, 6 5/8 REG \\
\hline & & 9 & Stabilizer & 32 & 979.7 & 17.375 & 2.813 & 180.59 & $\begin{array}{l}\text { Integral Blade Stabilizer } 17 \text { 3/8" FG, } 83 / 4 x \\
3 \text { in, } 6 \text { 5/8 REG }\end{array}$ \\
\hline & & 10 & Sub & 3 & 982.7 & 9.5 & 2.5 & 149.91 & Float Sub $8,8 \times 2$ 13/16 in, 6 5/8 REG \\
\hline & & 11 & Mud Motor & 26.3 & 1009 & 17.25 & 7.85 & 188.21 & PDM 9-5/8" 5/6 3.0 ST, 9 5/3" in, 6 5/8 REG \\
\hline & & 12 & Bit & 1 & 1010 & 17.5 & & 565 & PDC Bit, $3 \times 16,0,589$ in2 \\
\hline
\end{tabular}

Nota: La figura 6, muestra las herramientas necesarias para perforar la sección superficial del pozo planeado hasta una profundidad de 1010 pies.

Fuente: Autores mediante WellPlan, Landmark R500.

Figura 7.

Ensamblaje sección 12 1/4" @ 5100 pies, diseñado para el pozo HRSS-1

\begin{tabular}{|c|c|c|c|c|c|c|c|c|}
\hline & & Section Type & $\begin{array}{c}\text { Length } \\
\text { (ft) }\end{array}$ & $\begin{array}{l}\text { Measured } \\
\text { Depth (ft) }\end{array}$ & OD (in) & ID (in) & $\begin{array}{l}\text { Weight } \\
\text { (ppf) }\end{array}$ & Item Description \\
\hline \multirow[t]{14}{*}{$\bar{z}$} & - & Drill Pipe & 4160 & 4160 & 5 & 4.276 & 21.44 & Drill Pipe 5 in, 19.50 ppf, S, NC50(XH), P \\
\hline & At 0 & Heavy Weight & 91 & 4251 & 5 & 3 & 49.7 & Heavy Weight Drill Pipe, 5 in, 49.70 ppf \\
\hline & & Jar & 32.34 & 4283.3 & 6.5 & 2.75 & 91.79 & Hydraulic Jar Dailey Hyd., 6 1/2 in \\
\hline & & Heavy Weight & 540 & 4823.3 & 5 & 3 & 49.7 & HwDP, 5.000 in, $41 / 2$ NC 50 \\
\hline & & Sub & 2.67 & 4826 & 6.562 & 3 & 147 & Cross Over, 6.562 in, 4 1/2 NC50 \\
\hline & & Drill Collar & 185.9 & 5011.9 & 6.5 & 2.875 & 75.06 & Drill Collar, 6.500 IN, 75.06 ppf, 6 5/8 NC 46 \\
\hline & & Sub & 3 & 5014.9 & 6.25 & 3 & 147 & Cross Over, 6.250 in, $65 / 8$ NC46 \\
\hline & & Drill Collar & 30 & 5044.9 & 8.1 & 3.25 & 158.57 & Non-Mag Drill Collar, 8.100 in $65 / 8$ REG \\
\hline & 7 & Sub & 1.57 & 5046.5 & 8.57 & 6 & 147 & Cross Over, 8.570 in, $65 / 8$ REG \\
\hline & 1 & MWD & 25.19 & 5071.7 & 8.21 & 6 & 141.11 & MWD Tool, $8.210 \mathrm{in}, 141.11 \mathrm{ppf}, 65 / 8^{n} \mathrm{FH}$ \\
\hline & H & Sub & 1.67 & 5073.3 & 8.57 & 3.188 & 147 & Cross Over, 8.570 in, $65 / 8 \mathrm{FH}$ \\
\hline & & Drill Collar & 12 & 5085.3 & 8.25 & 2 & 169.3 & Non-Mag Drill Collar $81 / 4$ in, 2 in, 4 5/8 REG \\
\hline & & Stabilizer & 13.66 & 5099 & 9.125 & 3.75 & 190.84 & $\begin{array}{l}\text { Steerable Stabilizer, } 9.125 \text { in, } 190.84 \text { ppf, } 6 \\
\text { 5/8" REG }\end{array}$ \\
\hline & & Bit & 1 & 5100 & 12.25 & & 267 & Polycrystalline Diamond Bit, $3 \times 16,0.589 \mathrm{in}^{2}$ \\
\hline
\end{tabular}

Nota: La figura 7, muestra las herramientas necesarias para perforar la sección superficial del pozo planeado hasta una profundidad de 5100 pies.

Fuente: Autores mediante WellPlan, Landmark R500. 
Figura 8. Ensamblaje sección 12 1/4" @ 6340 pies, diseñado para el pozo HRSS-1

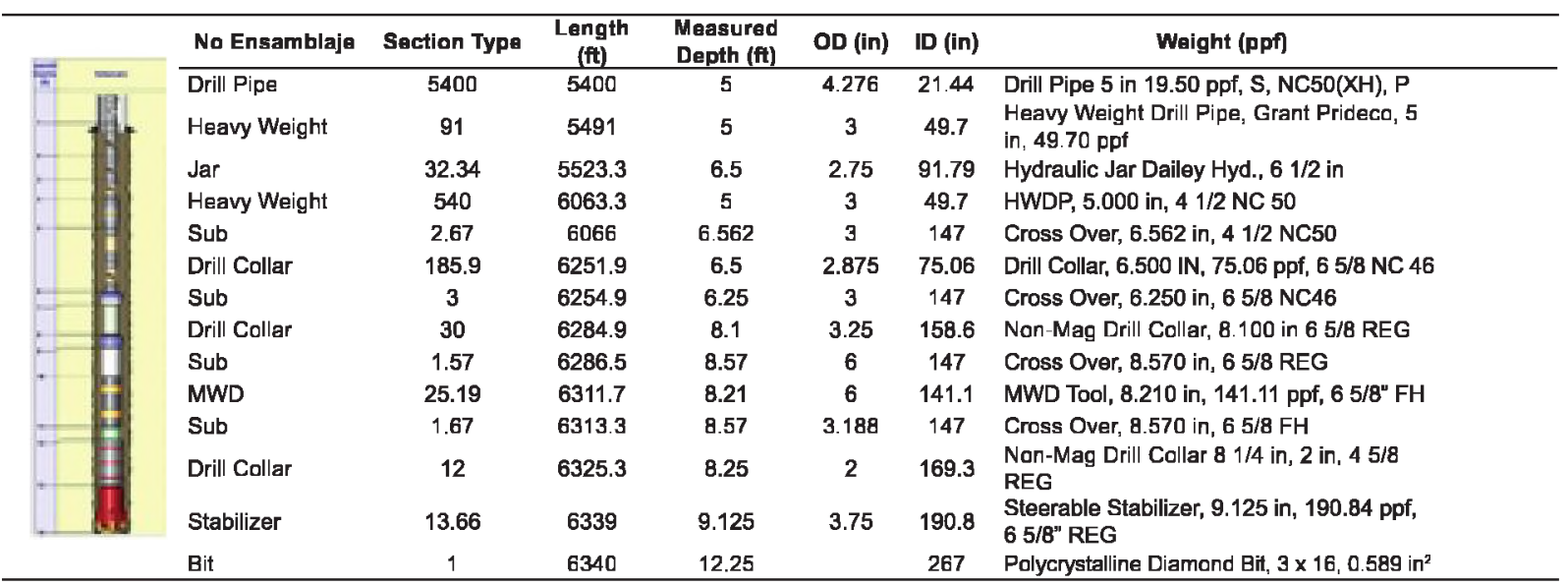

Nota: La figura 8, muestra las herramientas necesarias para perforar la sección superficial del pozo planeado hasta una profundidad de 6340 pies.

Fuente: Autores mediante WellPlan, Landmark R500.

Figura 9. Ensamblaje sección 8 1/2", diseñado para el pozo HRSS-1

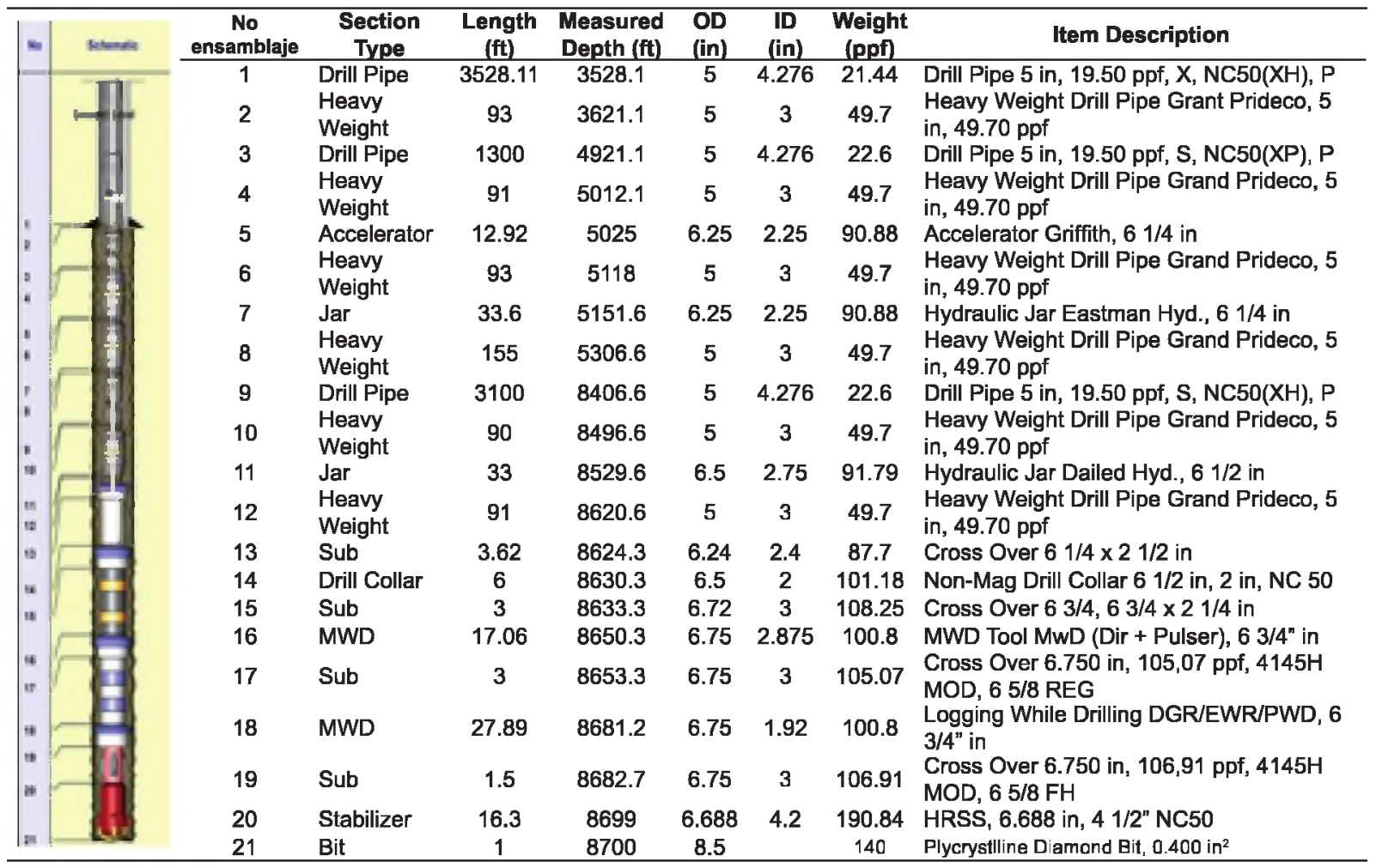

Nota: La figura 9, muestra la sección diseñada para aplicar los HRSS con el fin de generar con ángulo de construcción de $8 \% 100 \mathrm{Ft}$, en esta sección se logra geonavegar a lo largo de 1700 pies por lo cual es necesario el uso de martillos tanto en tensión como en compresión.

Fuente: Autores mediante WellPlan, Landmark R500. 
LIINEA DE INVESTIGACIÓN: NUEVAS TECNOLOGIAS DE PERFORACIÓN

De la sección 17 1/2", podemos destacar técnicamente los siguientes aspectos:

\subsection{Sección 12 1/4"@ 5100 Ft}

El estudio de ingeniería demuestra la viabilidad técnica para realizar la primera fase de la sección intermedia de 12 1/4"@ 5100 Ft de profundidad en el cual se manteien una velocidad de perforación promedio de $256 \mathrm{ft} / \mathrm{hr}$, en el cual no se supera las limitantes técnicos de operación que se permite para la perforación de un pozo hasta la profundidad de $5100 \mathrm{Ft}$, vale anotar, la adición de RSS en esta sección, con martillos y tuberías pesadas, aumentando en la resistencia del torque de toda la tubería perforada. De esta sección podemos destacar:

\subsection{Sección 12 1/4"@6340 Ft}

Esta sección se presenta con la continuación de la sección de 12 1/4" después de realizar un viaje de acondicionamiento a @5100 Ft, De esta sección podemos destacar:

\subsection{Sección 8 1/2"}

Esta sección es perforada con alto angulo de construcción, con más de 1700 pies de geonavegación en las arenas de la formación $\mathrm{K} 1$, en esta sección se usó el sistema rotativo direccional híbrido, el resultado de esta sección permitió comprobar técnicamente la aplicabilidad del uso de la herramienta HRSS. De esta sección podemos destacar:

\section{Referencias}

Ahmed, U. Optimized Shale Resource Development: Balance Between Technology and Economic Considerations Introduction: The conceptual balance between technology and cost 2014.

Amghar, Y. IADC/SPE 151283 PDC Bit Steerability Modeling and Testing for Push-the-bit and Pointthe-bit RSS; 2012. 1-12.

Archer, Powerdrive. High Build Rate RSS Delivers Dogleg Assurance in Challenging Formations.

Avery, Michael. SPE / IADC 119446 High Angle Directional Drilling With 95 / 8-in. Casing in Offshore Qatar. 2009. 1-10.

\section{Comentarios}

El uso de la herramienta direccional de Sistemas Rotativos Direccionales Hibridos permite profundizar 610 Ft el KOP en la sección de 12\%", según el programa de perforación, aumentando la velocidad de perforación de $40 \mathrm{Ft} / \mathrm{hr}$ a $50 \mathrm{Ft} / \mathrm{hr}$ en la fase de $81 / 2$ " lo que representa un aumento de ROP del $24 \%$, en términos generales el diseño del pozo HRSS-1 permite reducir el $33 \%$ del tiempo (4 días) total de perforación con referencia al pozo de referencia Cast-451.

Al llevar acabo la perforación con la herramienta HRSS es posible pagar un valor máximo de $\$ 639,483.41$ USD, de tal manera que para hacer económicamente viable la perforación con herramienta HRSS el precio de la perforación direccional debe estar entre el rango de la perforación direccional convencional y la perforación direccional HRSS que son $\$ 286,245.48$ USD y $\$ 639,483.41$ USD respectivamente.

La utilización de los sistemas RSS híbridos es de gran ayuda para la secciones de alta inclinación ya que éstos permiten direccionar el pozo con rotación continua, lo que permite reducir efectos negativos como lo es el fenómeno stick slip y problemas en limpieza de hoyo lo que puede provocar una futura pega de tubería, al disminuir los tiempos de perforación de 4 días, se obtiene un ahorro de $\$ 351,292.77$ USD. 
Bardisi, Tareq Al. Hybrid High Build Rate RSS Improves Challenging Directional Control in a Soft Abrasive Drilling Environment in Oman Drilling Operation 2014. 1-9.

Company, Amoco Production. A Method for Designing a Complex Directional Drilling Program Applied in Cook Inlet, Alaska. 1981.

Dupuis, C. SPE-169206-MS Avoid Pilot Holes, Land Wells, and Optimize Well Placement and Production with Deep Directional Resistivity Logging-While-Drilling 2014.

Herrington, David. New Technology Enhances Rotary Steerable System performance and Provides Superior Borehole Quality and Reduces Vibration in Rotary Steerable Applications; 2013, Society of Petroleum Engineers.

Maidla, Eric. SPE / IADC 92558 Field Proof of the New Sliding Technology for Directional Drilling, 2005.

Park, Byeolteo. Hybrid 4-Pad Rotary Steerable System for Directional Drilling of U nconventional Resources; 2013.

Park, Jieun. Design and Analysis of a Rotary Steerable System Using a New Hybrid Steering for Directional Drilling, 2013.

Shehab, A. SPE 167651. Innovative Drilling Technology Reduced Drilling Time in Deep Drilling Jurassic 16in Hole Section-Case Study BHA / Bit Drilling Challenges; 2013. 